\title{
A KKM-RESULT AND AN APPLICATION FOR BINARY AND NON-BINARY CHOICE FUNCTIONS
}

\section{Juan V. Llinares, M. Carmen Sánchez and Begoña Subiza}

WP-AD 2000-04

Correspondence to J.V. Llinares: Universidad de Murcia. Facultad de Economía y Empresa. Departamento de Fundamentos del Análisis Económico. Campus de Espinardo. 30100 Murcia. Telf.: +34 968 363737; Fax: +34 968 363750; e-mail: llinares@fcu.um.es.

Editor: Instituto Valenciano de Investigaciones Económicas, s.a.

First Edition February 2000

Depósito Legal: V-506-2000

IVIE working papers offer in advance the results of economic research under way in order to encourage a discussion process before sending them to scientific journals for their final publication.

* The authors wish to thank J.E. Peris for his helpful comments. The work of J.V. Llinares has been partially supported by a fellowship grant from the Programa Nacional de Becas FPI en el Extranjero, Ministerio de Educación y Cultura (Spain). The work of M.C. Sánchez has been partially supported by a TMR fellowship grant of the EU, contract No. ERBFMBICT 97 2668. These authors also acknowledge financial support from the Spanish DGICYT under project PB98-0553. The work of B. Subiza has been partially supported by the Spanish DGICYT under project PB97-0120. Financial support from the Instituto Valenciano de Investigaciones Económicas (I.V.I.E.) is also acknowledged.

** J.V. Llinares \& M.C. Sánchez: University of Murcia; B. Subiza: University of Alicante. 


\title{
A KKM-RESULT AND AN APPLICATION FOR BINARY AND NON-BINARY CHOICE FUNCTIONS
}

\author{
Juan V. Llinares, M. Carmen Sánchez \& Begoña Subiza
}

A B S T R A C T

By generalizing the classical Knaster-Kuratowski-Mazurkiewicz Theorem, we obtain a result that provides sufficient conditions to ensure the non-emptiness of several kinds of choice functions. This result generalizes well-known results on the existence of maximal elements for binary relations (Bergstrom, 1975; Walker, 1977; Tian, 1993), on the non-emptiness of nonbinary choice functions (Nehring, 1996; Llinares and Sánchez, 1999) and on the non-emptiness of some classical solutions for tournaments (top cycle and uncovered set) on non-finite sets.

KEYWORDS: Binary Choice Function; Non-Binary Choice Function; KKM Theorem; Maximal Elements; Tournament Solutions. 


\section{Introduction}

An interesting problem in choice theory consists of finding sufficient conditions to ensure the non-emptiness of choice functions on a class of choice situations sufficiently large, especially whenever infinite sets of alternatives are considered. This kind of result is very important, not only in economics but also in decision analysis, optimization and game theory. In general, there are two different approaches to dealing with this problem, those of binary and non-binary choice functions.

In the binary case, the choice function is made by using a binary relation (representing preferences), and the choice set consists of the best elements according to this binary relation. There is a great number of works devoted to the analysis of the existence of maximal elements, since they are considered "the best ones". In order to ensure the existence of maximal elements in each feasible subset, the acyclicity of the binary relation is a necessary condition; but there are many contexts in which to ask, a priori, for acyclicity of the binary relation is too strong a restriction. This is the case of realistic social decision mechanisms as, for instance, majority voting where it is well known that cycles may appear. This fact has inspired many papers on the problem of choosing the best elements when there are no maximal elements; the study of tournaments (asymmetric and complete binary relations), in particular, has been extensively developed (see for instance Miller, 1977, 1980 and McKelvey, 1986).

The non-binary approach considers situations in which the choice function is not representable by a binary relation. Some of the motivations for considering this kind of choice function are the unresolvedness of preferences (see Nehring, 1997) or the use of non-classical mechanisms (see Aizerman and Malishevski, 1981). To our best knowledge, the only results that can be found in the literature that ensure the non-emptiness of non-binary choice functions are those presented by Nehring (1996), who analyzes this problem for a class of contraction-consistent choice functions, and that of LLinares and Sánchez (1999), who solve the same problem by using weaker conditions and by enlarging the family of sets on which the non-emptiness can be ensured.

The aim of this work is to provide a result on the non-emptiness of choice functions that unifies all these different approaches considered in the literature. Since most of them are based on a reasoning of non-empty intersection, we start by generalizing the classical Knaster-Kuratowski-Mazurkiewicz Theorem (from now on, KKM Theorem) to a context in which no linear structure is required. Then, as a consequence, a result on the non-emptiness of choice functions is presented. This result can be applied to the different choice functions mentioned above (maximal elements, choices from tournaments and non-binary choice functions). 


\section{A KKM result}

Let $X$ be a topological space and $\mathcal{F}(X)$ the family of non-empty finite subsets of $X$. For each $B \subseteq X$, its topological closure is denoted by $\operatorname{cl}(B)$ and, if $A \subseteq X$ and $B \subseteq A, c l_{A}[B]$ denotes the topological closure of $B$ on $A$ with respect to the relative topology induced on $A$ by the topology of $X$. Moreover, if $X$ is a topological vector space, the convex hull of $B \subseteq X$ is denoted by $H(B)$. As usual, $\Delta_{n}$ is the $n$-dimensional simplex of $\mathbb{R}^{n+1}$ (i.e., $\Delta_{n}=H\left(\left\{e_{i}: i=0,1, \ldots, n\right\}\right)$ with $\left\{e_{i}\right\}_{i=0}^{n}$ the canonical basis of $\left.\mathbb{R}^{n+1}\right)$ and, for all $J \subseteq\{0,1, \ldots, n\}, \Delta_{J}$ represents the corresponding face (i.e., $\left.\Delta_{J}=H\left(\left\{e_{i}: i \in J\right\}\right)\right)$.

We are going to present our generalized KKM result by making use of a general abstract convexity structure called L-structure which is a powerful tool to ensure the existence of maximal elements (see LLinares, 1998a). Formally,

Definition 1 (Ben-El-Mechaiekh et al., 1998) Let $X$ be a topological space, $X$ has an $\boldsymbol{L}$-structure if there exists a non-empty valued correspondence $\Psi: \mathcal{F}(X) \rightarrow X$ and for all $B \in \mathcal{F}(X)$, namely $B=\left\{b_{0}, b_{1}, \ldots, b_{n}\right\}$, there exists a continuous function $f^{B}: \Delta_{n} \rightarrow \Psi(B)$ such that for all $J \subseteq\{0,1, \ldots, n\}, f^{B}\left(\Delta_{J}\right) \subseteq \Psi\left(\left\{b_{i}: i \in J\right\}\right)$.

The pair $(X, \Psi)$ is called an $\mathbf{L}$-space.

Remark 1 The notion of an L-space is equivalent to that of an mc-space (LLinares 1998b), which generalizes the notion of usual convexity as well as other abstract convexity structures (simplicial convexity, c-spaces,...). For instance, if $X$ is a topological vector space, then an L-structure on $X$ can easily be defined by considering $\Psi: \mathcal{F}(X) \rightarrow X, \Psi(B)=H(B)$ for all $B=\left\{b_{0}, b_{1}, \ldots, b_{n}\right\} \in \mathcal{F}(X)$, and by defining $f^{B}: \Delta_{n} \rightarrow \Psi(B)$ as $f^{B}(\lambda)=\sum_{i=0}^{n}$ $\lambda_{i} b_{i}$, for all $\lambda=\left(\lambda_{0}, \lambda_{1}, \ldots, \lambda_{n}\right) \in \Delta_{n}$. So, in Definition 1 , function $f^{B}$ can be interpreted, in a sense, as being an abstract convex combination of the elements in $B$.

The classical KKM Theorem (see for instance Border, 1985) provides a sufficient condition for a finite family of closed subsets of $\mathbb{R}^{n}$ to have non-empty intersection.

Theorem 1 [Knaster-Kuratowski-Mazurkiewicz Theorem] Let $\left\{F_{0}, F_{1}, \ldots, F_{n}\right\}$ be a family of closed subsets of $\Delta_{n}$ such that for every $J \subseteq\{0,1, \ldots, n\}$ we have $\Delta_{J} \subseteq \cup_{i \in J} F_{i}$. Then, $\bigcap_{i=0}^{n} F_{i}$ is compact and non-empty. 
We provide a similar result but in the more general context of L-spaces. To do so, we need the notion of generalized KKM-correspondence, that extends the notion of KKM-correspondence introduced by Dugundji and Granas in the context of usual convexity (Dugundji and Granas, 1982; Definition II.5.11) to the context of L-spaces.

Definition 2 Let $X$ and $Y$ be topological spaces such that $X$ has an $L$ structure defined by $\Psi: \mathcal{F}(X) \rightarrow X$ and by $f^{B}: \Delta_{n} \rightarrow \Psi(B)$ for each $B \in \mathcal{F}(X)$. A correspondence $\Gamma: Y \rightarrow X$ is said to be a generalized $\boldsymbol{K} \boldsymbol{K} M$-correspondence, if for all $\left\{y_{0}, y_{1}, \ldots, y_{n}\right\} \in \mathcal{F}(Y)$, there exists a subset $B=\left\{x_{0}, x_{1}, \ldots, x_{n}\right\} \in \mathcal{F}(X)$, such that for all $J \subseteq\{0,1, \ldots, n\}$, it is satisfied that

$$
f^{B}\left(\Delta_{J}\right) \subseteq \cup_{j \in J} \Gamma\left(y_{j}\right) .
$$

With respect to continuity conditions, we make use of a weak continuity notion called Transfer Closedness. This condition has been used by Zhou and Tian (1992) and by Tian (1993), among others, to obtain the existence of maximal elements.

Definition 3 [Transfer closedness] Let $X$ and $Y$ be topological spaces. A correspondence $\Phi: Y \rightarrow X$ is said to be transfer closed-valued on $Y$ if for every $y \in Y$ and $x \in X$, if $x \notin \Phi(y)$ then there exists $y^{\prime} \in Y$ such that $x \notin \operatorname{cl}\left[\Phi\left(y^{\prime}\right)\right]$.

Theorem 2 Let $X$ and $Y$ be topological spaces and $\Gamma: Y \rightarrow X$ a transfer closed-valued correspondence on $Y$ such that there exists $y^{*} \in Y$ with $c l\left[\Gamma\left(y^{*}\right)\right]$ compact. Then, the following conditions are equivalent:

i) There exists an L-structure on $X$ such that $\Gamma$ is a generalized KKMcorrespondence.

ii) $\bigcap_{y \in Y} \Gamma(y) \neq \emptyset$.

Proof. $[\mathbf{i}) \Rightarrow$ ii)] Assume that there exists an L-structure on $X$ given by $\Psi: \mathcal{F}(X) \rightarrow X$ and by $f^{B}: \Delta_{n} \rightarrow \Psi(B)$ for each $B \in \mathcal{F}(X)$, such that $\Gamma$ is a generalized KKM-correspondence. Since $\Gamma$ is transfer-closed valued on $Y$, it is easy to show that the following equity holds,

$$
\bigcap_{y \in Y} \Gamma(y)=\bigcap_{y \in Y} c l[\Gamma(y)] .
$$

So, it is sufficient to prove that $\underset{y \in Y}{\cap} c l[\Gamma(y)]$ is non-empty. To do so, we first prove that the family $\{c l[\Gamma(y)]\}_{y \in Y}$ satisfies the non-empty finite intersection property. Consider $\left\{y_{0}, y_{1}, \ldots, y_{n}\right\} \in \mathcal{F}(Y)$ and $\left\{c l\left[\Gamma\left(y_{i}\right)\right]\right\}_{i=0}^{n}$. Since $\Gamma: Y \rightarrow$ $X$ is a generalized KKM-correspondence, we know that, associated to this 
set $\left\{y_{0}, y_{1}, \ldots, y_{n}\right\}$, there exists a subset $B=\left\{x_{0}, x_{1}, \ldots, x_{n}\right\} \in \mathcal{F}(X)$, such that for all $J \subseteq\{0,1, \ldots, n\}$, it is satisfied that

$$
f^{\bar{B}}\left(\Delta_{J}\right) \subseteq \cup_{j \in J} \Gamma\left(y_{j}\right) \subseteq \cup_{j \in J} c l\left[\Gamma\left(y_{j}\right)\right] .
$$

So, if we define $R_{i}=\left(f^{B}\right)^{-1}\left[c l\left[\Gamma\left(y_{i}\right)\right]\right]$ for all $i \in\{0,1, \ldots, n\}$, then we know that $\left\{R_{i}\right\}_{i=0}^{n}$ is a family of closed subsets of $\Delta_{n}$ and, by (1), that $\Delta_{J} \subseteq \cup_{j \in J} R_{j}$ for all $J \subseteq\{0,1, \ldots, n\}$. So, we can apply Theorem 1 to obtain that there exists $z \in \bigcap_{i=0}^{n} R_{i}$, that is, $f^{B}(z) \in \bigcap_{i=0}^{n} c l\left[\Gamma\left(y_{i}\right)\right]$ and we can conclude that family $\{c l[\Gamma(y)]\}_{y \in Y}$ has the non-empty finite intersection property. But this implies that family $\left\{\operatorname{cl}[\Gamma(y)] \cap \operatorname{cl}\left[\Gamma\left(y^{*}\right)\right]\right\}_{y \in Y}$, which is a family of closed subsets of the compact subset $c l\left[\Gamma\left(y^{*}\right)\right]$, also satisfies this property. So, by compactness, we can ensure that

and since we can rewrite

$$
\bigcap_{y \in Y}\left[c l[\Gamma(y)] \cap \operatorname{cl}\left[\Gamma\left(y^{*}\right)\right]\right] \neq \emptyset ;
$$

we obtain the required conclusion.

$$
\bigcap_{y \in Y}\left[c l[\Gamma(y)] \cap c l\left[\Gamma\left(y^{*}\right)\right]\right]=\bigcap_{y \in Y} c l[\Gamma(y)],
$$

$[\mathbf{i i}) \Rightarrow \mathbf{i})]$ Conversely, if we assume that $\bigcap_{y \in Y} \Gamma(y) \neq \emptyset$, then we can take $x^{*} \in \bigcap_{y \in Y} \Gamma(y)$ and define an L-structure on $X$ as follows: $\Psi: \mathcal{F}(X) \rightarrow X$ is given by the constant function $\Psi(B)=x^{*}$ for all $B=\left\{b_{0}, b_{1}, \ldots, b_{n}\right\} \in \mathcal{F}(X)$, and function $f^{B}: \Delta_{n} \rightarrow \Psi(B)$ is defined as $f^{B}(\lambda)=x^{*}$ for all $\lambda \in \Delta_{n}$. Then it is easy to verify that, with this L-structure, $\Gamma$ is a generalized KKMcorrespondence.

The previous result generalizes the classical KKM Theorem, since it has been stated for a non-finite family of not necessarily closed subsets in a general topological space and no sort of linear structure has been required. Furthermore, it also generalizes most of the KKM results obtained within the usual convex framework (Fan's Lemma in Dugundji and Granas, 1982; or Lemma 1 in Tian, 1993), as well as those obtained in other abstract convexity structures (Bielawski, 1987; or Horvath, 1991).

\section{An application to binary and non-binary choice functions}

In this section we present a general result on the non-emptiness of choice functions. Throughout this section, $X$ denotes a topological space and $\mathfrak{D}$ a family of non-empty subsets of $X$ that represents the different feasible sets presented for choice. Given $A \in \mathfrak{D}, \mathfrak{D}_{A}$ denotes a family of non-empty subsets of $A$. A choice function is a correspondence that assigns to each choice situation a subset of it, that is, 
$C: \mathfrak{D} \rightarrow X$ such that $C(A) \subseteq A \quad$ for all $A \in \mathfrak{D}$.

Theorem 3 Let $X$ be a topological space and $C: \mathfrak{D} \rightarrow X$ a choice function. If for $A \in \mathfrak{D}$ there exists a family $\mathfrak{D}_{A}$ and a correspondence $\Omega_{A}: \mathfrak{D}_{A} \rightarrow A$ satisfying that:

i) $\underset{D \in \mathfrak{D}_{A}}{\cap} \Omega_{A}(D) \subseteq C(A)$;

ii) $\Omega_{A}$ is transfer closed-valued;

iii) $A$ is an $L$-space such that $\Omega_{A}$ is a generalized KKM-correspondence;

iv) there exists $D^{*} \in \mathfrak{D}_{A}$ with $c_{A}\left[\Omega_{A}\left(D^{*}\right)\right]$ compact on $A$;

then, $C(A) \neq \emptyset$.

Proof. It is sufficient to apply Theorem 2 to any $A \in \mathfrak{D}$ satisfying all of the hypothesis of Theorem 3, to ensure that $\underset{D \in \mathfrak{D}_{A}}{\cap} \Omega_{A}(D) \neq \emptyset$. Then, by applying i), the conclusion is obtained.

In order to show that this result generalizes some results on the nonemptiness of usual choice functions (maximal elements, top cycle, uncovered set, non-binary choice functions,...), we consider the family $\mathfrak{D}_{A}=\mathcal{F}(A)$ for every $A \in \mathfrak{D}$ and, given the choice function $C: \mathfrak{D} \rightarrow X$, we define correspondence $\Omega_{A}: \mathcal{F}(A) \rightarrow A$ as follows,

$$
\Omega_{A}(T)=\{a \in A \mid a \in C(T \cup\{a\})\} \text { for all } T \in \mathcal{F}(A) .
$$

In order to present the results in a clear way, we analyze the case of binary and that of non-binary choice functions in two different subsections.

\subsection{Binary choice functions}

\subsubsection{Existence of maximal elements}

The notion of a binary choice function requires that the choice depends on a binary relation $R$. The most usual case in this context is that in which the choice is made by maximizing this binary relation. We consider $R$ a complete and reflexive binary relation, so this choice function can be expressed as $C: \mathfrak{D} \rightarrow X$ such that for all $A \in \mathfrak{D}, C(A)=\left\{a^{*} \in A \mid a^{*} R a\right.$ $\forall a \in A\}$. Therefore, the non-emptiness choice is reduced to ensure the existence of greatest (or maximal) elements. It is also possible to analyze the binary case through non-complete preference relations, but since both approaches have been proven to be equivalent (Kim and Richter, 1986) we shall consider the weak approach. 
In this context, there are two basic ways to prove the existence of maximal elements on compact sets. One of them assumes convexity and continuity conditions (see Fan, 1961; Sonnenschein, 1971; and Shafer and Sonnenschein, 1975 among others), while the other approach assumes transitivity and continuity conditions (see Bergstrom, 1975; Walker, 1977; Campbell and Walker, 1990; or Subiza and Peris, 1997). Among the most recent work, we must mention the results of Zhou and Tian (1992), Tian (1993) or LLinares (1998a) that unify these two approaches. One of the most general works that provides sufficient conditions to ensure the existence of maximal elements is that of Tian (1993), so we shall focus on his results. He considers Hausdorff topological vector spaces and imposes transfer closedness as well as a convexity condition (transfer FS-convexity), to the upper contour set correspondence $\left(U^{A}: A \rightarrow A, U^{A}(a)=\{b \in A \mid b R a\}\right)$.

Definition 4 [Transfer FS-convexity] Let $Y$ be a topological space and $X$ a non-empty convex subset in a topological vector space $E$. A correspondence $\Phi: Y \rightarrow X$ is said to be transfer FS-convex on $Y$ if for any $\left\{y_{0}, y_{1}, \ldots, y_{n}\right\} \in \mathcal{F}(Y)$, there exists a corresponding subset $\left\{x_{0}, x_{1}, \ldots, x_{n}\right\} \in$ $\mathcal{F}(X)$ such that for any $J \subseteq\{0,1, \ldots, n\}$, we have

$$
H\left(\left\{x_{j}: j \in J\right\}\right) \subseteq \cup_{j \in J} \Phi\left(y_{j}\right) .
$$

$A$ binary relation $R$ defined on $X$ is said to be transfer FS-convex on $A$ if the correspondence $U^{A}: A \rightarrow A$ is transfer FS-convex on $A$.

Next we prove that Tian's result on the existence of greatest elements (Tian, 1993) can be obtained as a consequence of Theorem 3.

Corollary 1 [Tian, 1993; Theorem 1] Let A be a non-empty compact convex subset of a Hausdorff topological vector space $E$ and let $R_{A}$ be a binary relation defined on $A$ such that $U^{A}: A \rightarrow A$ is transfer closed-valued on $A$. If $R_{A}$ is transfer FS-convex on $A$, then the set of greatest elements of $R_{A}$ on $A$ is non-empty and compact.

Proof. It is sufficient to consider $\mathfrak{D}$ as the family of non-empty compact convex subsets of $E$ satisfying the conditions of the corollary. To apply Theorem 3, we define the choice correspondence, $C: \mathfrak{D} \rightarrow E$ such that for all $A \in \mathfrak{D}, C(A)=\left\{a^{*} \in A \mid a^{*} R_{A} a \quad \forall a \in A\right\}$. Moreover, for all $A \in \mathfrak{D}$ we consider $\mathfrak{D}_{A}=\mathcal{F}(A)$. Note that for all $T \in \mathcal{F}(A), \Omega_{A}(T)=\bigcap_{a \in T} U^{A}(a)$ which is transfer closed-valued and obviously $\underset{T \in \mathfrak{F}(A)}{\cap} \Omega_{A}(T)=C(A)$, so conditions i) and ii) from Theorem 3 are satisfied. Condition iv) is also satisfied since $A$ is a compact set, so for all $D \in \mathfrak{D}_{A}, \operatorname{cl}_{A}\left[\Omega_{A}(D)\right]$ is compact on $A$. In order to show that $A$ is an L-space such that $\Omega_{A}: \mathcal{F}(A) \rightarrow A$ is a generalized KKM-correspondence we define the L-structure on $A$ as follows. For all 
$B=\left\{b_{0}, b_{1}, \ldots, b_{n}\right\} \in \mathcal{F}(A)$, correspondence $\Psi: \mathcal{F}(A) \rightarrow A$ is given by $\Psi(B)=A$ and function $f^{B}: \Delta_{n} \rightarrow \Psi(B)$ is defined as a constant function given by the last element, that is, $f^{B}(\lambda)=b_{n}$ for all $\lambda \in \Delta_{n}$. So, it is obviously a continuous function and, for all $J \subseteq\{0,1, \ldots, n\}$, it is satisfied that $f^{B}\left(\Delta_{J}\right)=b_{n} \in \Psi\left(\left\{b_{j}: j \in J\right\}\right)=A$.

Finally, we verify that $\Omega_{A}: \mathcal{F}(A) \rightarrow A$ is a generalized KKM-correspondence for this L-structure. To do so, first note that for all $T \in \mathcal{F}(A)$, since $U^{A}$ is transfer FS-convex, we can apply Theorem 2 by considering the usual convexity to ensure that $\bigcap_{t \in T} U^{A}(t) \neq \emptyset$. So, for all $\left\{T_{0}, T_{1}, \ldots, T_{n}\right\} \subseteq \mathcal{F}(A)$, we consider the family of subsets $\left\{\bigcup_{j=0}^{i} T_{j}\right\}_{i=0}^{n}$ and we apply the previous reasoning to select $t_{i} \in \cap U^{A}(t)$. Thus, we define subset $B=\left\{t_{0}, t_{1}, \ldots, t_{n}\right\} \in$ $t \in \stackrel{\cup}{j}=0_{i} T_{j}$

$\mathcal{F}(A)$ and denote $T^{*}=\bigcup_{i=0}^{n} T_{i}$, therefore

$$
f^{B}\left(\Delta_{J}\right)=t_{n} \in \bigcap_{t \in T^{*}} U^{A}(t)=\bigcap_{i=0}^{n} \underset{t \in T_{i}}{\cap} U^{A}(t)=\bigcap_{i=0}^{n} \Omega_{A}\left(T_{i}\right) \subseteq \cup_{j \in J}^{\cup} \Omega_{A}\left(T_{j}\right),
$$

and we can conclude that $\Omega_{A}$ is a generalized KKM-correspondence and Theorem 3 can be applied to ensure that $C(A) \neq \emptyset$.

We would like to mention that, by a similar reasoning and as a consequence of Theorem 3, the other results in Tian's work (1993), on the existence of maximal elements for strict binary relations and for the case in which non-compact and non-convex choice sets are considered, can be also obtained.

\subsubsection{Tournament Solutions}

Miller (1977) showed that majority preferences may be represented as a tournament. A tournament $\mathbb{T}=(X, P)$ consists of a non-empty set $X$ and a complete asymmetric binary relation $P$ defined on $X$ ( $x P y$ means $x$ beats $y$ ). Typically, in such situations, there is no Condorcet winner (an alternative which beats all others), but there are other, arguably compelling, methods of formulating non-empty choice functions based on majority preferences. Some central methods are the top cycle (Schwartz 1972, 1986 and Miller 1977) and the uncovered set (Fishburn 1977 and Miller 1980). Given a tournament $\mathbb{T}=(X, P)$, if $P^{\infty}$ denotes the transitive closure of $P$ on $X\left(x P^{\infty} y\right.$ $\Leftrightarrow$ there are $x_{1}, x_{2}, \ldots, x_{n} \in X$ such that $x=x_{1} P x_{2}, x_{2} P x_{3}, \ldots, x_{n-1} P x_{n}=$ $y)$, the top cycle can be defined as $T C(X, P)=\left\{x \in X \mid x P^{\infty} y\right.$ for all $y \in X, x \neq y\}$, whereas an alternative $x$ is in the uncovered set $U C(X, P)$, if for all $y \in X, x P y$ or there is $z \in X$ such that $x P z$ and $z P y$ (two step principle).

By using Theorem 3 we obtain the following results which ensure the non-emptiness of the above mentioned choice functions for general sets of 
alternatives.

Corollary 2 Let $A$ be a non-empty compact subset of a Hausdorff topological space $E$ and let $\mathbb{T}=(A, P)$ be a tournament such that $U_{\infty}^{A}: A \rightarrow A$, $U_{\infty}^{A}(a)=\left\{b \in A: b P^{\infty} a\right\} \cup\{a\}$ is transfer closed-valued on $A$. Then $T C(A, P) \neq \emptyset$.

Proof. Consider the choice function $C: \mathfrak{D}_{A} \rightarrow X$ defined as $C(T)=$ $\left\{a \in T \mid a P^{\infty} t\right.$ for all $\left.t \in T, t \neq a\right\}$, being $P^{\infty}$ the transitive closure of $P$ on $A$. As we have defined $\Omega_{A}(T)=\{a \in A \mid a \in C(T \cup\{a\})\}$ for all $T \in \mathcal{F}(A)$, then $\underset{T \in \mathcal{F}(A)}{\bigcap} \Omega_{A}(T)=T C(A, P)=\bigcap_{a \in A} U_{\infty}^{A}(a)$, and since $U_{\infty}^{A}$ is transfer closed-valued, $\Omega_{A}$ is transfer closed-valued, so conditions i) and ii) from Theorem 3 are satisfied. In order to prove that $\Omega_{A}: \mathcal{F}(A) \rightarrow A$ is a generalized KKM-correspondence, we define the L-structure on $A$ by considering $\Psi: \mathcal{F}(A) \rightarrow A$ given by $\Psi(B)=A$ for all $B \in \mathcal{F}(A)$. For any $B=\left\{b_{0}, b_{1}, \ldots, b_{n}\right\} \in \mathcal{F}(A)$, we know that there exists $b^{*} \in B$ such that $b^{*} P^{\infty} b_{j}$ for all $b_{j} \in B, b^{*} \neq b_{j}$. The function $f^{B}: \Delta_{n} \rightarrow \Psi(B)$ is then defined as a constant function given by this element (if there is more than one of these elements, we then choose the one with greatest index according to the order fixed in subset $B$ ), that is, $f^{B}(\lambda)=b^{*}$ for all $\lambda \in \Delta_{n}$. So, it is obviously a continuous function and, for all $J \subseteq\{0,1, \ldots, n\}$, it is satisfied that $f^{B}\left(\Delta_{J}\right)=b^{*} \in \Psi\left(\left\{b_{j}: j \in J\right\}\right)=A$. But then, $\Omega_{A}$ : $\mathcal{F}(A) \rightarrow A$ is a generalized KKM-correspondence because we can define, for all $\left\{T_{0}, T_{1}, \ldots, T_{n}\right\} \subseteq \mathcal{F}(A)$, the subset $B=\left\{t_{0}, t_{1}, \ldots, t_{n}\right\} \in \mathcal{F}(A)$, where $t_{i} P^{\infty} t$ for all $t \in T_{i}, t \neq t_{i}$ and we select, by reasoning as above, the element $t^{*} \in B$ such that $t^{*} P^{\infty} t_{j}$ for all $t_{j} \in B, t^{*} \neq t_{j}$. Then we can conclude that $t^{*} P^{\infty} t$ for all $t \in T^{*}=\bigcup_{i=0}^{n} T_{i}, t^{*} \neq t$ and

$$
f^{B}\left(\Delta_{J}\right)=t^{*} \in \bigcap_{i=0}^{n} \Omega_{A}\left(T_{k}\right) \subseteq \cup_{j \in J} \Omega_{A}\left(T_{j}\right) .
$$

Finally, note that the last condition in Theorem 3 is obviously satisfied due to the fact that $A$ is a compact set. Theorem 3 can, therefore, be applied to conclude that $C(A)=T C(A, P) \neq \emptyset$.

Corollary 3 Let $A$ be a non-empty compact subset of a Hausdorff topological space $E$ and $\mathbb{T}=(A, P)$ a tournament such that $U_{2}^{A}: A \rightarrow A$, $U_{2}^{A}(a)=\{b \in A: b P a$ or $b P c, c P a$ for some $c \in A\} \cup\{a\}$ is transfer closed-valued on $A$. Then $U C(A, P) \neq \emptyset$.

Proof. The proof is omitted since it is analogous to the previous one.

\subsection{Non-binary choice functions}

We start by introducing the different assumptions that are used in the nonbinary context to prove the non-emptiness of choice functions. As usual, 
we consider that $\mathfrak{D}$ is a family of non-empty subsets of $X$ that contains all of the finite subsets of $X$ (see Moulin, 1985; Nehring, 1996; LLinares and Sánchez, 1999). Since the result obtained by LLinares and Sánchez (1999) is more general than that of Nehring (1996), we shall focus on their result for the non-binary case. The assumptions imposed to the choice function are the following,

Non-emptiness: For all $A \in \mathcal{F}(X), C(A) \neq \emptyset$.

Axiom $\alpha^{*}:$ For all $A \in \mathcal{F}(X)$, there exists $a^{*} \in C(A)$ such that for all $B \in \mathcal{F}(A)$, if $a^{*} \in B$ then $a^{*} \in C(B)$.

Finitariness: For all $A \in \mathfrak{D}$, if $a \in A$ and for all $B \in \mathcal{F}(A),[a \in B \Rightarrow$ $a \in C(B)]$, then $a \in C(A)$.

Transfer Continuity: For all $A \in \mathfrak{D}, \Omega_{A}$ is a transfer closed-valued correspondence.

We start by proving that the contraction consistency assumption, Axiom $\alpha^{*}$, implies that it is possible to define an L-structure on the space such that $\Omega_{A}$ is a generalized KKM-correspondence.

Lemma 1 Let $X$ be a topological space and $C: \mathfrak{D} \rightarrow X$ a choice function satisfying Axiom $\alpha^{*}$. Then for all $A \in \mathfrak{D}$ it is possible to define an $L$ structure on $A$ such that $\Omega_{A}$ is a generalized KKM-correspondence.

Proof. Given $A \in \mathfrak{D}$, we define the L-structure on $A$ by considering $\Psi$ : $\mathcal{F}(A) \rightarrow A$ given by $\Psi(B)=A$ for all $B=\left\{b_{0}, b_{1}, \ldots, b_{n}\right\} \in \mathcal{F}(A)$. To define function $f^{B}: \Delta_{n} \rightarrow \Psi(B)$ we apply Axiom $\alpha^{*}$ to subset $B$ and we select the corresponding element of $B$ which is always chosen in any subset of $B$ that contains it, namely $b^{*}$ (if there is more than one of these elements, then we choose the one with greatest index according to the order fixed in subset $B$ ). Then, function $f^{B}$ is defined as a constant function given by this element, that is, $f^{B}(\lambda)=b^{*}$ for all $\lambda \in \Delta_{n}$. So, it is obviously a continuous function and for all $J \subseteq\{0,1, \ldots, n\}$ it is satisfied that $f^{B}\left(\Delta_{J}\right)=b^{*} \in \Psi\left(\left\{b_{j}: j \in\right.\right.$ $J\})=A$. Finally, it is easy to verify that $\Omega_{A}: \mathcal{F}(A) \rightarrow A$ is a generalized KKM-correspondence. To do so, define, for all $\left\{T_{0}, T_{1}, \ldots, T_{n}\right\} \subseteq \mathcal{F}(A)$, subset $B=\left\{t_{0}, t_{1}, \ldots, t_{n}\right\} \in \mathcal{F}(A)$, where $t_{i}$ is one of the elements provided when Axiom $\alpha^{*}$ is applied to subset $\bigcup_{j=0}^{i} T_{j}$. But since $t_{n}$ is the element provided by Axiom $\alpha^{*}$ whenever subset $\bigcup_{i=0}^{n} T_{i}$ is considered, we know that $t_{n} \in \bigcap_{i=0}^{n} \Omega_{A}\left(T_{i}\right)$; so, for any $J \subseteq\{0,1, \ldots, n\}$, we have,

$$
f^{B}\left(\Delta_{J}\right)=t_{n} \in \cap_{i=0}^{n} \Omega_{A}\left(T_{i}\right) \subseteq \bigcup_{j \in J} \Omega_{A}\left(T_{j}\right) .
$$


Corollary 4 [LLinares and Sánchez, 1999] Let X be a topological space and $C: \mathfrak{D} \rightarrow X$ a choice function satisfying Non-emptiness, Axiom $\alpha^{*}$, Finitariness and Transfer Continuity, then for all $A \in \mathfrak{D}$ such that there exists $T^{*} \in \mathcal{F}(A)$ with $c_{A}\left[\Omega_{A}\left(T^{*}\right)\right]$ compact, $C(A) \neq \emptyset$.

Proof. Consider $A \in \mathfrak{D}$ such that there exists $T^{*} \in \mathcal{F}(A)$ with $c l_{A}\left[\Omega_{A}\left(T^{*}\right)\right]$ compact. It is then clear that Finitariness implies condition i) from Theorem 3 , transfer continuity implies ii), Lemma 1 implies iii) and iv) is obviously satisfied by the hypothesis. We can therefore, apply Theorem 3 to obtain the required conclusion. 


\section{References}

M.A. Aizerman and A.V. Malishevski, General theory of best variant choice: some aspects, IEE Transactions on Automatic Control 26 (1981) 1030-1040.

H.S. Ben-El-Mechaiekh, S. Chebbi, M. Florenzano and J.V. LLinares, Abstract convexity and fixed points, Journal of Mathematical Analysis and Applications 222 (1998) 138-150.

T.C. Bergstrom, Maximal elements of acyclic relations on compact sets, Journal of Economic Theory 10 (1975) 403-404.

R. Bielawski, Simplicial convexity and its applications, Journal of Mathematical Analysis and Applications 127 (1987) 155-171.

K. Border, Fixed points theorems with applications to economics and game theory (Cambridge University Press, 1985).

D.E. Campbell and M. Walker, Maximal elements of weakly continuous relations, Journal of Economic Theory 50 (1990) 459-464.

J. Dugundji and A. Granas, Fixed Point Theory, vol.1 (Polish scientific publishers, Warszawa, 1982).

K. Fan, A generalization of Tychonoff's fixed point theorem, Math. Ann. 142 (1961) 305-310.

P. Fishburn, Condorcet social choice functions, SIAM Journal of Applied Mathematics 33 (1977) 469-489.

Ch. Horvath, Contractibility and generalized convexity, Journal of Mathematical Analysis and Applications 156 (1991) 341-357.

T. Kim and M.K. Richter, Nontransitive-nontotal consumer theory, Journal of Economic Theory 38 (1986) 324-363.

J.V. LLinares, Unified treatment of the problem of the existence of maximal elements in binary relations. A characterization, Journal of Mathematical Economics 29 (3) (1998a) 285-302.

J.V. LLinares, Abstract convexity, some relations and applications, Working Paper CEPREMAP Couverture Orange n ${ }^{o} 9803$ (1998b).

J.V. LLinares and M.C. Sánchez, Non-binary choice functions on noncompact sets, Economics Letters 63 (1999) 29-32.

R. McKelvey, Covering, dominance, and institution-free properties of social choice, American Journal of Political Science 30 (1986) 283-314. 
N. Miller, Graph-theoretical approaches to voting, American Journal of Political Science 21 (1977) 769-803.

N. Miller, A new solution set for tournaments and majority voting: Further graph-theoretical appproaches to the theory of voting, American Journal of Political Science 24 (1980) 68-96.

H. Moulin, Choice functions over a finite set: A summary, Social Choice and Welfare 2 (1985) 147-160.

K. Nehring, Maximal elements of non-binary choice functions on compact sets, Economics Letters 50 (1996) 337-340.

K. Nehring, Rational choice and revealed preference without binariness, Social choice and Welfare 14 (1997) 403-425.

W. Shafer and H. Sonnenschein, Equilibrium in abstract economies without ordered preferences, Journal of Mathematical Economics 2 (1975) 345-348.

H. Sonnenschein, Demand theory without transitive preferences, with application to the theory of competitive equilibrium, in: J.S.Chipman, L.Hurwicz, M.K. Richter and H. Sonnenschein eds., Preference, Utility and Demand, (Harcourt Brace Jovanovich, New York, 1971) pp. 215223.

B. Subiza and J.E. Peris, Numerical representation for lower quasicontinuos preferences, Mathematical Social Sciences 33 (1997) 149-156.

T. Schwartz, Rationality and the myth of the maximum, No $\widehat{u s} 6$ (1972) $97-117$.

T. Schwartz, The Logic of Collective Action (New York, Columbia University Press, 1986).

G. Tian, Necessary and sufficient conditions for maximization of a class of preference relations, Review of Economic Studies 60 (1993) 949-958.

M. Walker, On the existence of maximal elements, Journal of Economic Theory 16 (1977) 470-474.

J. Zhou and G. Tian, Transfer method for characterizing the existence of maximal elements of binary relations on compact or noncompact sets, SIAM J. Optimization 2 (1992) 360-375. 